\title{
BMJ Open Serum fibrinogen and cardiovascular events in Chinese patients with type 2 diabetes and stable coronary artery disease: a prospective observational study
}

\author{
Sheng-Hua Yang, Ying Du, Yan Zhang, Xiao-Lin Li, Sha Li, Rui-Xia Xu, \\ Cheng-Gang Zhu, Yuan-Lin Guo, Na-Qiong Wu, Ping Qing, Ying Gao, \\ Chuan-Jue Cui, Qian Dong, Jing Sun, Jian-Jun Li
}

To cite: Yang S-H, Du Y, Zhang Y, et al. Serum fibrinogen and cardiovascular events in Chinese patients with type 2 diabetes and stable coronary artery disease: a prospective observational study. BMJ Open 2017;7:e015041. doi:10.1136/ bmjopen-2016-015041

- Prepublication history and additional material are available. To view these files please visit the journal online (http://dx.doi. org/10.1136/bmjopen-2016015041).

Received 7 November 2016 Revised 6 April 2017 Accepted 6 April 2017

\section{CrossMark}

Division of Dyslipidemia, State Key Laboratory of Cardiovascular Disease, Fuwai Hospital, National Center for Cardiovascular Diseases, Chinese Academy of Medical Sciences, Peking Union Medical College, Beijing, China

Correspondence to

Professor Jian-Jun Li; lijianjun938@126.com

\section{ABSTRACT}

Objectives The aim of this study was to investigate the association of serum fibrinogen with cardiovascular events (CVE) in Chinese patients with type 2 diabetes mellitus (T2DM) and stable coronary artery disease (CAD).

Design An observational study.

Setting FuWai Hospital in Beijing, China.

Participants A cohort of 1466 patients with T2DM and angiographic-proven stable CAD was evaluated. Outcome measures Baseline serum fibrinogen levels were measured and trisected into 'low', 'middle' and 'high'. Their association with CVE was explored using Cox proportional hazard models.

Results With 20.2 months (average) follow-up, 44 (3\%) were lost to follow-up and 96 patients developed CVE. Compared with the patients without CVE, the ones who developed CVE had higher levels of fibrinogen. Univariable regression revealed a significant relation of fibrinogen to CVE (HR (HR) 1.25, 95\% Cl 1.06 to 1.47, p=0.010) per SD increase of fibrinogen at baseline. After adjusting for multiple established cardiovascular disease (CVD) risk factors, the association persisted ( $\mathrm{HR} 1.30,95 \% \mathrm{Cl} 1.02$ to 1.66, $p=0.037$ ). Moreover, after adjusting for CVD risk factors, the HRs for middle-serum and high-serum fibrinogen concentration, using 'low' group as reference, were $1.23(95 \% \mathrm{Cl} 0.69$ to 2.20$)$ and $2.20(95 \% \mathrm{Cl} 1.11$ to $3.36, p=0.049$ ).

Conclusions We first indicated that elevated fibrinogen level was independently associated with increased CVE in Chinese patients with T2DMand stable CAD.

\section{INTRODUCTION}

Fibrinogen, as a marker of thrombosis and inflammation, is associated with cardiovascular diseases (CVD). It has been demonstrated that elevated fibrinogen level is significantly associated with intima-media thickness and subclinical atherosclerosis. ${ }^{1-5}$ Also, previous study revealed that fibrinogen independently predicted future ischaemic stroke risk and incident hypertension. ${ }^{6-8}$ What is more, fibrinogen

\section{Strengths and limitations of this study}

- We, for the first time, reported that baseline fibrinogen was associated with cardiovascular events (CVEs) in Chinese patients with type 2 diabetes (T2DM) and stable coronary artery disease (CAD).

- The number of CVEs was relatively small and the follow-up duration was relatively short, so further study is needed.

- We just investigated whether the one-time baseline level of fibrinogen was a predictor of short-term outcome in patients withT2DM and stable CAD.

has been well established to be a strong and independent cardiovascular risk factor in general population. ${ }^{8-12}$ Data suggested that baseline plasma fibrinogen level could predict cardiovascular events (CVE) in general individuals and non-diabetics. ${ }^{391013}$

It is worthy of mentioning that individuals with type two diabetes mellitus (T2DM) have higher levels of plasma fibrinogen compared with those without T2DM. ${ }^{8}$ The most common cause for mortality in patients with $\mathrm{DM}$ is coronary artery disease (CAD). Thereby, it is logically hypothesised that hyperfibrinogenaemia could contribute to increase CVE in this disease. However, studies on the role of fibrinogen in cardiovascular outcomes in diabetes are both limited and controversial. $^{615-17}$

Based on such situation and combined with a fact that no data are currently available so far with regard to the relationship between fibrinogen and CVE in patients with T2DM and stable $\mathrm{CAD}$, we, therefore, performed a prospective observational study of the association of baseline plasma fibrinogen and the risk of CVE in patients with diabetes and stable CAD. 


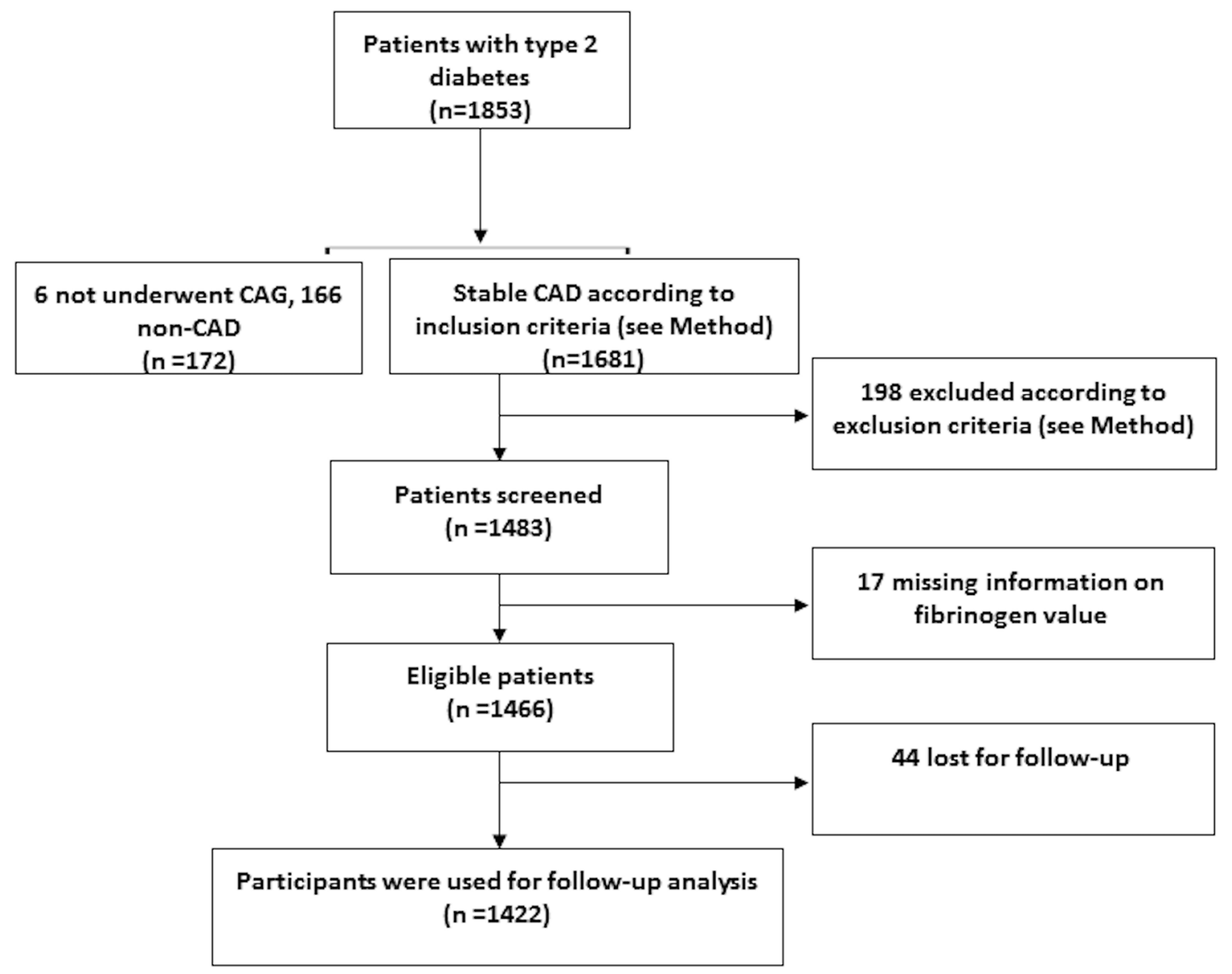

Figure 1 Flow chart of the study. CAD, coronary artery disease.

Patients and methods

Study population

In this study, consecutive patients hospitalised in our division were screened between April 2011 and July 2015 for the following inclusion criteria: over the age of 18 years, T2DM and angiographic-proven stable CAD. Exclusion criteria were acute coronary syndrome (ACS), in-hospital cardiac death at first admission in our division, unavailable data of baseline fibrinogen concentration, the existence of any infectious or systematic inflammatory diseases, significant haematologic disorders, thyroid dysfunction, severe liver insufficiency (transaminases $>10$ times upper reference levels) and/or severe renal insufficiency (end-stage renal disease or chronic dialysis treatment) and malignant tumours. According to the inclusion and exclusion criteria, a total of 1466 eligible patients were enrolled (figure 1). The study was performed according to the Declaration of Helsinki, and the hospital ethics review board (Fuwai Hospital and National Center for Cardiovascular Diseases, Beijing, China) approved the protocol. All the subjects gave written informed consent.

\section{Biochemical analysis and baseline examination}

Overnight fasting blood samples were drawn from each patient at admission and centrifuged at room temperature within 2 hours. As described in our previous studies, ${ }^{18} 19$ the plasma levels of fibrinogen were quantitatively measured by the method of Clauss and a Stagoauto analyser with STA Fibrinogen kit (Diagnostic Stago, Taverny, France). Glucose, haemoglobinA1c (HbAlc), uric acid and lipid profile were determined by standard methods. The concentrations of high-sensitivity C-reactive protein (hs-CRP) were determined using immunoturbidimetry (Beckmann Assay 360, Bera, California, USA).

Height and weight were measured, and body mass index (BMI) $\left(\mathrm{kg} / \mathrm{m}^{2}\right)$ was calculated. DM was defined as a fasting plasma glucose $\geq 126 \mathrm{mg} / \mathrm{dL}(7.0 \mathrm{mmol} / \mathrm{L})$ in multiple determinations and/or the current use of medication for diabetes. Hypertension was defined as repeated blood pressure measurements $\geq 140 / 90 / 90 \mathrm{~mm} \mathrm{Hg}$ (at least two times in different environments) or currently taking antihypertensive drugs. Stable CAD was defined as typical angina-like chest pain brought on by exertion and relieved by rest or sublingual nitrates or both, a positive treadmill exercise test ( $>1 \mathrm{~mm}$ ST-segment depression) and stable obstructive lesion $>50 \%$ in at least one of the three major coronary arteries or major branches assessed by at least two independent senior interventional cardiologists who had no knowledge of the patients' clinical characteristics and biochemical results. Dyslipidaemia was considered to be present in patients if they had fasting total cholesterol $\geq 200 \mathrm{mg} / \mathrm{dL}$ or triglyceride $\geq 150 \mathrm{mg} / \mathrm{dL}$. 
Definition of events and follow-up

The primary outcomes were CVE. CVE were defined as the cardiac death, stroke, non-fatal myocardial infarction (MI), postdischarge revascularisation (percutaneous coronary intervention (PCI)/coronary artery bypass grafting (CABG)) due to clinical deterioration or unstable angina (UA).The follow-up data collection was performed by the trained nurses or cardiologists who were blinded to the aim of this study by means of standardised telephone interviews at 6-month intervals. Follow-up time was calculated as the number of months from the enrolment up to the last traceable hospital outpatient or inpatient record or telephone interview before March 2016, and was censored on the date of

Table 1 Characteristics of the study cohort with and without cardiovascular events of myocardial infarction, cardiac death, myocardial revascularisation and fatal or non-fatal stroke

\begin{tabular}{|c|c|c|c|}
\hline & CVE $(n=96)$ & No CVE $(n=1326)$ & p-Value \\
\hline Age (years) & $58.54 \pm 10.65$ & $59.29 \pm 9.81$ & 0.473 \\
\hline Body mass index $\left(\mathrm{kg} / \mathrm{m}^{2}\right)$ & $26.32 \pm 3.07$ & $26.22 \pm 3.21$ & 0.790 \\
\hline Gender, men, \% (n) & $70.8(68)$ & 70.7 (938) & 0.984 \\
\hline Dyslipidaemia, \% (n) & $88.5(85)$ & $80.6(1068)$ & 0.055 \\
\hline Hypertension, \% (n) & $78.1(75)$ & 72.9 (966) & 0.265 \\
\hline Current smoking, \% (n) & $56.3(54)$ & $51.2(679)$ & 0.343 \\
\hline Duration of diabetes (years) & $10.24 \pm 6.66$ & $8.64 \pm 6.50$ & 0.151 \\
\hline Previous PCl, \% (n) & $22.9(22)$ & $24.0(318)$ & 0.810 \\
\hline Previous CABG, \% (n) & $1.0(1)$ & $3.4(45)$ & 0.208 \\
\hline Family history of CAD, \% (n) & $17.7(17)$ & $14.8(195)$ & 0.224 \\
\hline Systolic blood pressure $(\mathrm{mm} \mathrm{Hg})$ & $124.94 \pm 14.64$ & $127.86 \pm 16.41$ & 0.117 \\
\hline Diastolic blood pressure (mm Hg) & $74.93 \pm 9.12$ & $77.01 \pm 10.17$ & 0.071 \\
\hline Left ventricle ejection fraction (\%) & $60.4 \pm 10.2$ & $63.7 \pm 8.0$ & $<0.001$ \\
\hline Total cholesterol (mmol/L) & $4.10 \pm 1.16$ & $4.10 \pm 1.21$ & 0.962 \\
\hline HDL-cholesterol (mmol/L) & $0.98 \pm 0.28$ & $1.03 \pm 0.27$ & 0.126 \\
\hline LDL-cholesterol (mmol/L) & $2.44 \pm 0.99$ & $2.46 \pm 0.96$ & 0.827 \\
\hline Triglycerides (mmol/L) & $1.79(1.28,2.53)$ & $1.56(1.18,2.21)$ & 0.054 \\
\hline Apo A1 (g/L) & $1.36 \pm 0.37$ & $1.32 \pm 0.28$ & 0.140 \\
\hline Apo B (g/L) & $0.99 \pm 0.34$ & $0.94 \pm 0.33$ & 0.113 \\
\hline $\mathrm{HbA1c}(\%)$ & $7.89 \pm 1.54$ & $7.50 \pm 1.33$ & 0.007 \\
\hline Glucose (mmol/L) & $7.52 \pm 2.68$ & $7.18 \pm 2.28$ & 0.155 \\
\hline hs-CRP, mg/L & $1.83(1.08,4.47)$ & $1.54(0.77,3.23)$ & 0.017 \\
\hline Uric acid $(\mu \mathrm{mol} / \mathrm{L})$ & $368.39 \pm 96.44$ & $340.26 \pm 93.36$ & 0.005 \\
\hline eGFR (mL/min) & $82.03 \pm 24.55$ & $80.12 \pm 25.58$ & 0.471 \\
\hline Fibrinogen (g/L) & $3.52 \pm 0.85$ & $3.30 \pm 0.8$ & 0.015 \\
\hline \multicolumn{4}{|l|}{ Medication } \\
\hline Statin, \% (n) & $69.8(67)$ & 71.9 (953) & 0.707 \\
\hline Aspirin, \% (n) & $88.1(84)$ & $86.7(1149)$ & 0.794 \\
\hline Beta-blocker, \% (n) & $52.4(50)$ & $48.2(639)$ & 0.601 \\
\hline Calcium channel blocker & $11.5(11)$ & $20.7(275)$ & 0.166 \\
\hline ACEI/ARB & $30.0(29)$ & $26.5(351)$ & 0.521 \\
\hline OADs, \% (n) & $48.9(47)$ & $54.3(720)$ & 0.296 \\
\hline Insulin, \% (n) & $27.1(26)$ & $25.8(342)$ & 0.813 \\
\hline OADs+insulin, \% (n) & $13.5(13)$ & $19.9(264)$ & 0.215 \\
\hline
\end{tabular}

Data are presented as mean \pm SD, median (25th, 75th percentiles) or \% (n).

p-values $<0.05$ in bold.

ACEI/ARB, angiotensin-converting enzyme inhibitor/angiotensin II receptor blocker; Apo, apolipoprotein; CVE, cardiovascular events; eGFR, estimated glomerular filtration rate; HbA1c, glycosylated haemoglobin type A1C; HDL, high-density lipoprotein; hs-CRP, high-sensitivity C-reactive protein; LDL, low-density lipoprotein; OADs, oral antidiabetic drugs. 
Table 2 Baseline characteristics of the cohort of the diabetic patients with stable CAD according to serum fibrinogen levels at baseline

\begin{tabular}{|c|c|c|c|c|}
\hline \multirow[b]{2}{*}{ Variables } & \multicolumn{3}{|c|}{ Fibrinogen tertiles ( $g / L)$} & \multirow[b]{2}{*}{ p-Value } \\
\hline & Low $(\leq 2.91)(n=476)$ & Middle $(2.91-3.51)(n=476)$ & High $(\geq 3.51)(n=470)$ & \\
\hline Age (years) & $58.65 \pm 9.66$ & $59.16 \pm 9.69$ & $59.91 \pm 10.22$ & 0.142 \\
\hline BMI $\left(\mathrm{kg} / \mathrm{m}^{2}\right)$ & $26.21 \pm 3.08$ & $26.18 \pm 2.92$ & $26.30 \pm 3.58$ & 0.838 \\
\hline Gender, men, \% (n) & $78.4(373)$ & $68.5(326)$ & $65.3(307)$ & $<0.001$ \\
\hline Dyslipidaemia, \% (n) & $80.5(383)$ & $81.1(385)$ & $81.9(385)$ & 0.848 \\
\hline Hypertension, \% (n) & $71.4(340)$ & 72.0 (342) & 76.4 (359) & 0.170 \\
\hline Current smoking, \% (n) & $53.2(253)$ & $53.3(253)$ & $48.3(227)$ & 0.219 \\
\hline $\mathrm{SBP}(\mathrm{mm} \mathrm{Hg})$ & $126.82 \pm 15.45$ & $127.57 \pm 17.08$ & $127.58 \pm 17.04$ & 0.723 \\
\hline $\mathrm{DBP}(\mathrm{mm} \mathrm{Hg})$ & $76.60 \pm 10.00$ & $77.07 \pm 10.26$ & $76.64 \pm 10.15$ & 0.731 \\
\hline $\mathrm{TC}(\mathrm{mmol} / \mathrm{L})$ & $3.86 \pm 1.05$ & $4.17 \pm 1.17$ & $4.27 \pm 1.24$ & $<0.001$ \\
\hline $\mathrm{HDL}-\mathrm{C}(\mathrm{mmol} / \mathrm{L})$ & $1.02 \pm 0.25$ & $1.03 \pm 0.26$ & $1.03 \pm 0.30$ & 0.739 \\
\hline LDL-C (mmol/L) & $2.28 \pm 0.89$ & $2.52 \pm 0.94$ & $2.60 \pm 1.01$ & $<0.001$ \\
\hline Triglycerides (mmol/L) & $1.57(1.12,2.13)$ & $1.60(1.24,2.34)$ & $1.54(1.19,2.17)$ & 0.092 \\
\hline $\mathrm{HbA1C}(\%)$ & $7.23 \pm 1.23$ & $7.52 \pm 1.28$ & $7.84 \pm 1.46$ & $<0.001$ \\
\hline Glucose (mmol/L) & $6.81 \pm 2.10$ & $7.22 \pm 0.32$ & $7.58 \pm 2.43$ & $<0.001$ \\
\hline hs-CRP, mg/L & $0.88(0.52,1.45)$ & $1.54(0.82,2.71)$ & $3.64(1.83,8.34)$ & $<0.001$ \\
\hline Uric acid ( $\mu \mathrm{mol} / \mathrm{L})$ & $340.93 \pm 95.56$ & $339.06 \pm 86.97$ & $346.60 \pm 98.62$ & 0.441 \\
\hline CVD events, \% (n) & $5.0(24)$ & $5.7(27)$ & $9.6(45)$ & 0.011 \\
\hline Duration of diabetes (years) & $9.03 \pm 6.82$ & $8.89 \pm 6.03$ & $10.83 \pm 6.83$ & 0.356 \\
\hline Gensini score & $28(19,49)$ & $30(16,56)$ & $33(17,61)$ & 0.315 \\
\hline Fibrinogen $(\mathrm{g} / \mathrm{L})$ & $2.55 \pm 0.31$ & $3.21 \pm 0.16$ & $4.20 \pm 0.74$ & $<0.001$ \\
\hline
\end{tabular}

Data are presented as mean \pm SD, median (25th, 75th percentiles) or \% (n). CVD events: myocardial infarction, cardiac death, myocardial revascularisation and fatal or non-fatal stroke.

$\mathrm{BMI}$, body mass index; CAD, coronary artery disease; CVD, cardiovascular disease events: myocardial infarction, cardiac death, myocardial revascularisation and fatal or non-fatal stroke; DBP, diastolic blood pressure; HbA1c, glycosylated haemoglobin type A1C; HDL-C, highdensity lipoprotein cholesterol; hs-CRP, high-sensitivity C-reactive protein; LDL-C, low-density lipoprotein cholesterol; SBP, systolic blood pressure; TC, total cholesterol.

the first CVE. Death of a participant was reported by relatives or the general practitioner who treated the participant. Three experienced physicians who were blinded to any of the study data independently classified the events.

\section{Statistical analysis}

Continuous variables were presented as mean $\pm \mathrm{SD}$ or median (25th, 75th percentiles) and categorical variables as percentages. Fibrinogen levels were trisected into 'low' $(\leq 2.91 \mathrm{~g} / \mathrm{L})$, 'middle' $(2.91-3.51 \mathrm{~g} / \mathrm{L})$ and 'high' $(\geq 3.51 \mathrm{~g} / \mathrm{L})$. Comparisons of continuous baseline data between two or more groups were performed with Student t-test and analysis of variance, respectively, and $\chi^{2}$ test was used to compare categorical data. Kruskal-Wallis $\mathrm{H}$ test was used for non-normal variables. Test for linear trend (Jonckheere-Terpstra test) was performed by assigning median value for each tertile and treated as continuous variables.

Participants who were lost during follow-up were treated as censored observations and not used for follow-up analysis. Using the Cox proportional hazards model, HRs of CVE, with 95\% CIs were calculated using crude models as well as models adjusted for potential confounding factors. A $p<0.05$ for two-sided testing was considered statistically significant. The statistical analysis was performed with SPSS V.19.0.

\section{RESULTS}

\section{Baseline characteristics}

The baseline demographic, clinical characteristics and laboratory findings of the study cohort classified by outcome status (with CVE or not) were listed in table 1. Compared with the patients without CVE, the ones who developed CVE had lower left ventricle ejection fraction (LVEF) but higher levels of fibrinogen, HbA1c, hs-CRP and uric acid. Meanwhile, we found that there is a tendency to higher fasting triglyceride level in CVE group ( $p=0.054)$. In addition, there is no significant difference in age, BMI, gender, status of hypertension, smoking status and medical history between the patients with or without CVE.

Table 2 compared the baseline cardiovascular risk profiles of the study population, divided into 'low' $(\geq 2.91 \mathrm{~g} / \mathrm{L})$, 'middle' $(2.91-3.51 \mathrm{~g} / \mathrm{L})$ and 'high' 
$(\geq 3.51 \mathrm{~g} / \mathrm{L})$ subgroups on the basis of the fibrinogen concentration. The patients with middle and high levels of fibrinogen had higher percentage of CVE, female and higher levels of hs-CRP, total cholesterol, low-density lipoprotein cholesterol (LDL-C), HbA1c and fasting glucose compared with the 'low' group. No significant difference was found in age, BMI, blood pressure and smoking status.

\section{Outcomes}

The average follow-up time was 20.2 months (range from 0.4 to 49.3 ). During the follow-up period, 96 patients experienced cardiovascular event: $19(19.8 \%)$ non-fatal MIs, $55(57.3 \%)$ myocardial revascularisation procedures (PCI or $\mathrm{CABG}$ ) because of clinical deterioration, 15 (15.6\%) strokes and $7(7.3 \%)$ cardiac deaths. We calculated the number of new events per 100 person-years, namely, 2.0047/100 per 100 person-years. Patients who suffered ACS and underwent revascularisation procedures were assigned once in the analysis. Of the 1466 patients with diabetes and stable coronary artery disease, 44 (3\%) were lost to follow-up. Therefore, the data of 1422 participants were used for follow-up analysis.

\section{HRs of fibrinogen for study outcomes}

Univariable regression revealed significant association between serum fibrinogen level at baseline and CVE $(\mathrm{HR}=1.25,95 \%$ CI 1.06 to $1.47, \mathrm{p}=0.010)$ per SD increase of serum fibrinogen at baseline. After adjusting for multiple established CVD risk factors, the association persisted ( $\mathrm{HR}=1.30,95 \%$ CI 1.02 to $1.66, \mathrm{p}=0.037$ ) (table 3). Unadjusted HR for developing a CVE was significantly increased in the 'high' group compared
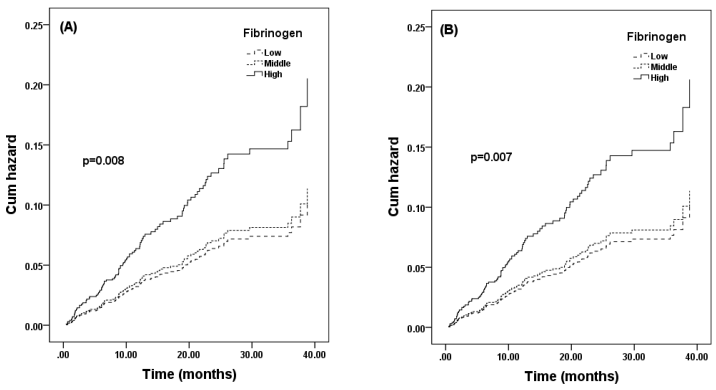

Figure 2 Unadjusted (A) and adjusted by sex and age (B) cumulative hazard of cardiovascular events based on the levels of fibrinogen (low, middle and high).

with the 'low' group at baseline, and similar results were also found after adjusting for sex and age (figure 2). After adjusting for established CVD risk factors, the HR for middle and high serum fibrinogen concentration, using 'low' group as reference, were 1.23 (95\% CI 0.69 to 2.20 ) and 2.20 (95\% CI 1.11 to 3.36) ( $\mathrm{p}$ for trend 0.049 ) (table 3 ).

\section{DISCUSSION}

In this prospective observational study in single centre with a relative large sample size, we, for the first time, found that baseline fibrinogen concentration was independently associated with CVE in Chinese patients with T2DM with stable CAD. Although the study might be limited by follow-up duration, the present data provided novel and important information with regard to the key issue whether the baseline fibrinogen concentration

Table 3 HRs for CVD events association with the plasma levels of fibrinogen (low, middle and high)

\begin{tabular}{|c|c|c|c|c|}
\hline & \multicolumn{3}{|c|}{1 SD fibrinogen increase } & p-Value \\
\hline Model 1 & \multicolumn{3}{|l|}{1.26 (1.06 to 1.49$)$} & 0.008 \\
\hline Model 3 & \multicolumn{3}{|l|}{1.36 (1.07 to 1.72$)$} & 0.011 \\
\hline Model 4 & \multicolumn{3}{|l|}{1.34 (1.05 to 1.71$)$} & 0.017 \\
\hline \multirow[b]{2}{*}{ Model } & \multicolumn{3}{|l|}{ HR $(95 \% \mathrm{Cl})$} & \multirow[b]{2}{*}{$p$ for trend } \\
\hline & Low (Reference) & Middle & High & \\
\hline Crude & 1.00 & $1.10(0.64$ to 1.91$)$ & $1.99(1.21$ to 3.26$)$ & 0.008 \\
\hline Model 1 & 1.00 & 1.10 (0.64 to 1.92$)$ & 2.00 (1.22 to 3.30$)$ & 0.007 \\
\hline Model 5 & 1.00 & 1.23 (0.69 to 2.20$)$ & $2.02(1.11$ to 3.68$)$ & 0.049 \\
\hline
\end{tabular}

Model 1: adjusted for sex and age; Model 2: Model 1+ total cholesterol, high-density lipoprotein cholesterol, low-density lipoprotein cholesterol, hypertension and smoking; Model 3: Model 2+ triglycerides, hs-CRP, and HbA1c; Model 4: Model 3+uric acid, body mass index and family history of CVD and Model 5: Model 4+left ventricle ejection fraction.

CVD, cardiovascular disease; HbA1c, glycosylated haemoglobin type A1C; hs-CRP, high sensitivity C-reactive protein. 
can be a marker for predicting the clinical outcomes in patients with T2DM and stable CAD.

Sufficient evidence has demonstrated that plasma fibrinogen is a risk factor for CVD in general population, ${ }^{368-10}$ but the role of fibrinogen in diabetes remains controversial. Some previous studies demonstrated that hyperfibrinogenaemia was an important risk factor for vascular complications in diabetes. ${ }^{20}$ For instance, a prospective cohort of 2329 patients with T1DM revealed that fibrinogen was significant and independent predictor of coronary heart disease in men. ${ }^{15}$ Moreover, increased fibrinogen level was shown to be associated with diabetic nephropathy in patients with T2DM. ${ }^{21} 22$ and T1DM. ${ }^{1523} 24$ Gargano Heart Study showed that fibrinogen was a predictor of incident major CVE after adjusting for sex, age, smoking habit and BMI status in patients with diabetes. ${ }^{25}$ In parallel, Bruno et al ${ }^{26}$ reported that fibrinogen had an independent predictive effect on cardiovascular mortality in T2DM. Furthermore, Stehouwer $e t a l^{27}$ revealed that baseline fibrinogen concentrations were strongly and independently associated with risk of all-cause death in patients with T2DM. On the contrary, some studies reported that the association between fibrinogen and incident $\mathrm{CAD}$ in patients with T2DM was non-significant via multivariable analyses. ${ }^{17} 28$ As we well known, the major cause of death in T2DM is macrovascular complications, especially CAD. Hence, in this study, we enrolled angiographic-proven CAD patients with T2DM and tried to examine the role of fibrinogen in such a study population of patients with T2DM and CAD. We found that elevated fibrinogen level at baseline was associated with increased CVE in patients with T2DM and stable CAD. In addition, we also found that fibrinogen was higher among those patients who developed a cardiovascular event compared with those who did not, which is in agreement with previous studies. ${ }^{9}{ }^{13}$ To our knowledge, this is the first time to investigate the relationship between fibrinogen and CVE in patients with T2DM and stable CAD.

Recently, the Action in Diabetes and Vascular Disease: Preterax and Diamicron Modified Release Controlled Evaluation (ADVANCE) study reported that baseline fibrinogen level was associated with an increased risk of macrovascular events and death in univariate analysis, but after further adjustment, fibrinogen was not an independent predictor of macrovascular. ${ }^{17}$ In this study, our finding is not consistent with the ADVANCE study, and we speculate that some reasons could explain it. First, the inclusive criteria are different: we chose the patients with T2DM and stable CAD, while the ADVANCE study chose the subjects with T2DM and a history of CVD (stroke, myocardial infarction, transient ischaemic attack, unstable angina, coronary or peripheral revascularisation, amputation, macroalbuminuria, proliferative retinopathy or photocoagulation, macular oedema or blindness in one eye) or one or more additional cardiovascular risk factors. Second, despite the patients from many countries in the ADVANCE study, Chinese and
Indians were excluded. Actually, there are ethnic differences in fibrinogen levels. ${ }^{29-32}$ Thus, whether the results also persist in Chinese is undetermined. Finally, the definitions of outcomes are different. In ADVANCE study, major macrovascular events were cardiovascular death, non-fatal myocardial infarction, or non-fatal stroke, while our cardiovascular events were defined as cardiac death, stroke, non-fatal MI, postdischarge revascularisation (PCI/CABG) due to clinical deterioration or UA. In addition, our previous cross-sectional study has revealed that plasma fibrinogen is associated with severity of CAD evaluated using Gensini score in patients with diabetes, ${ }^{19}$ which partly supports our present finding. Furthermore, in this study, we found that $3.515(\mathrm{~g} / \mathrm{L})$ is a cut-off value of fibrinogen to predict CVEs in our cohort, which is consistent with previous study. ${ }^{33}$

There were several limitations of this study. First, the number of events is relatively small to assess the prognostic value of fibrinogen. The conclusion needs to be testified by large-scale study in the future. Second, the follow-up duration was relatively short and longer follow-up will be required. Third, this sample population was collected prospectively from a single centre in China. Fibrinogen varies in race, ${ }^{29}$ so we do not know if our results would apply to other ethnic groups and studies from multiple centres are needed to test our finding. Finally, we just collected one-time baseline level of fibrinogen.

In conclusion, we found that fibrinogen was associated with CVE in Chinese patients with T2DM and stable $\mathrm{CAD}$. Further studies are required to clarify the issue completely.

Contributors S-HY analysed the data and drafted the manuscript. J-JL planned and designed the study. The rest of the authors were involved in collecting and researching data, reviewing and editing manuscript. All authors read and approved the final manuscript.

Funding This work was partially supported by Youth Foundation of Peking Union Medical College (3332016023), the National Natural Science Foundation of China (81070171, 81241121), the Specialized Research Fund for the Doctoral Program of Higher Education of China (20111106110013), the Capital Special Foundation of Clinical Application Research (Z121107001012015), the Capital Health Development Fund (2011400302) and the Beijing Natural Science Foundation (7131014) awarded to Dr Jian-Jun Li, MD, PhD.

Competing interests None declared.

\section{Patient consent Obtained.}

Ethics approval The study was performed according to the Declaration of Helsinki, and the hospital ethics review board (Fuwai Hospital and National Center for Cardiovascular Diseases, Beijing, China) approved the protocol.

Provenance and peer review Not commissioned; externally peer reviewed.

Data sharing statement The technical appendix, statistical code and data set are available from the corresponding author.

Open Access This is an Open Access article distributed in accordance with the Creative Commons Attribution Non Commercial (CC BY-NC 4.0) license, which permits others to distribute, remix, adapt, build upon this work non-commercially, and license their derivative works on different terms, provided the original work is properly cited and the use is non-commercial. See: http://creativecommons.org/ licenses/by-nc/4.0/

(C) Article author(s) (or their employer(s) unless otherwise stated in the text of the article) 2017. All rights reserved. No commercial use is permitted unless otherwise expressly granted. 


\section{REFERENCES}

1. Sabeti S, Exner M, Mlekusch W, et al. Prognostic impact of fibrinogen in carotid atherosclerosis: nonspecific indicator of inflammation or independent predictor of disease progression? Stroke 2005;36:1400-4.

2. Green D, Foiles N, Chan C, et al. Elevated fibrinogen levels and subsequent subclinical atherosclerosis: the CARDIA Study. Atherosclerosis 2009;202:623-31.

3. De Luca G, Verdoia M, Cassetti E, et al. High fibrinogen level is an independent predictor of presence and extent of coronary artery disease among Italian population. J Thromb Thrombolysis 2011;31:458-63.

4. Temelkova-Kurktschiev T, Koehler C, Henkel E, et al. Leukocyte count and fibrinogen are associated with carotid and femoral intimamedia thickness in a risk population for diabetes. Cardiovasc Res 2002;56:277-83.

5. Willeit P, Thompson SG, Agewall S, et al. Inflammatory markers and extent and progression of early atherosclerosis: meta-analysis of individual-participant-data from 20 prospective studies of the PROGIMT collaboration. Eur J Prev Cardiol 2016;23:194-205.

6. Chuang SY, Bai $\mathrm{CH}$, Chen WH, et al. Fibrinogen independently predicts the development of ischemic stroke in a taiwanese population: cvdfacts study. Stroke 2009;40:1578-84.

7. Shankar A, Wang JJ, Rochtchina E, et al. Positive association between plasma fibrinogen level and incident hypertension among men: population-based cohort study. Hypertension 2006;48:1043-9.

8. Danesh J, Lewington S, Thompson SG, et al. Plasma fibrinogen level and the risk of Major cardiovascular diseases and nonvascular mortality: an individual participant meta-analysis. JAMA 2005;294:1799-809.

9. Sinning JM, Bickel C, Messow CM, et al. Impact of C-reactive protein and fibrinogen on cardiovascular prognosis in patients with stable angina pectoris: the AtheroGene study. Eur Heart J 2006;27:2962-8.

10. Kaptoge S, Di Angelantonio E, Pennells L, et al. C-reactive protein, fibrinogen, and cardiovascular disease prediction. $N$ Engl J Med 2012;367:1310-20.

11. Park CS, Ihm SH, Yoo KD, et al. Relation between C-reactive protein, homocysteine levels, fibrinogen, and lipoprotein levels and leukocyte and platelet counts, and 10-year risk for cardiovascular disease among healthy adults in the USA. Am J Cardiol 2010;105:1284-8.

12. Danesh J, Collins R, Appleby $P$, et al. Association of fibrinogen, C-reactive protein, albumin, or leukocyte count with coronary heart disease: meta-analyses of prospective studies. JAMA 1998;279:1477-82.

13. Luc G, Bard JM, Juhan-Vague I, et al. C-reactive protein, interleukin-6, and fibrinogen as predictors of coronary heart disease: the PRIME study. Arterioscler Thromb Vasc Biol 2003;23:1255-61.

14. Barazzoni R, Zanetti M, Davanzo G, et al. Increased fibrinogen production in type 2 diabetic patients without detectable vascular complications: correlation with plasma glucagon concentrations. J Clin Endocrinol Metab 2000;85:3121-5.

15. Soedamah-Muthu SS, Chaturvedi N, Pickup JC, et al. Relationship between plasma sialic acid and fibrinogen concentration and incident micro- and macrovascular complications in type 1 diabetes. The EURODIAB prospective complications study (PCS). Diabetologia 2008;51:493-501.

16. Engström G, Stavenow L, Hedblad B, et al. Inflammation-sensitive plasma proteins, diabetes, and mortality and incidence of myocardial infarction and stroke: a population-based study. Diabetes 2003;52:442-7.

17. Lowe G, Woodward M, Hillis G, et al. Circulating inflammatory markers and the risk of vascular complications and mortality in people with type 2 diabetes and cardiovascular disease or risk factors: the ADVANCE study. Diabetes 2014;63:1115-23.

18. Zhang Y, Zhu CG, Xu RX, et al. Relation of circulating PCSK9 concentration to fibrinogen in patients with stable coronary artery disease. J Clin Lipidol 2014;8:494-500.

19. Hong LF, Li XL, Luo SH, et al. Association of fibrinogen with severity of stable coronary artery disease in patients with type 2 diabetic mellitus. Dis Markers 2014;2014:1-8.

20. Ganda OP, Arkin CF. Hyperfibrinogenemia. An important risk factor for vascular complications in diabetes. Diabetes Care 1992;15:1245-50.

21. Tessari P, Kiwanuka E, Barazzoni R, et al. Diabetic nephropathy is associated with increased albumin and fibrinogen production in patients with type 2 diabetes. Diabetologia 2006;49:1955-61.

22. Azad N, Agrawal L, Emanuele NV, et al. Association of PAl-1 and fibrinogen with diabetic retinopathy in the Veterans Affairs Diabetes Trial (VADT). Diabetes Care 2014;37:501-6.

23. Klein RL, Hunter SJ, Jenkins AJ, et al. Fibrinogen is a marker for nephropathy and peripheral vascular disease in type 1 diabetes: studies of plasma fibrinogen and fibrinogen gene polymorphism in the DCCT/EDIC cohort. Diabetes Care 2003;26:1439-48.

24. Polat SB, Ugurlu N, Yulek F, et al. Evaluation of serum fibrinogen, plasminogen, $\alpha 2$-anti-plasmin, and plasminogen activator inhibitor levels (PAI) and their correlation with presence of retinopathy in patients with type 1 DM. J Diabetes Res 2014;2014:1-6.

25. Pacilli A, De Cosmo S, Trischitta V, et al. Role of relationship between $\mathrm{HbA} 1 \mathrm{c}$, fibrinogen and $\mathrm{HDL}$-cholesterol on cardiovascular disease in patients with type 2 diabetes mellitus. Atherosclerosis 2013;228:247-8

26. Bruno G, Merletti F, Biggeri A, et al. Fibrinogen and AER are Major independent predictors of 11-year cardiovascular mortality in type 2 diabetes: the Casale Monferrato Study. Diabetologia 2005;48:427-34

27. Stehouwer CD, Gall MA, Twisk JW, et al. Increased urinary albumin excretion, endothelial dysfunction, and chronic lowgrade inflammation in type 2 diabetes: progressive, interrelated, and independently associated with risk of death. Diabetes 2002;51:1157-65.

28. Saito I, Folsom AR, Brancati FL, et al. Nontraditional risk factors for coronary heart disease incidence among persons with diabetes: the Atherosclerosis risk in communities (ARIC) Study. Ann Intern Med 2000;133:81-91.

29. Cook DG, Cappuccio FP, Atkinson RW, et al. Ethnic differences in fibrinogen levels: the role of environmental factors and the betafibrinogen gene. Am J Epidemiol 2001;153:799-806.

30. Albert MA. Inflammatory biomarkers, race/ethnicity and cardiovascular disease. Nutr Rev 2007;65:234-8.

31. Albert MA, Glynn RJ, Buring JE, et al. Relation between soluble intercellular adhesion molecule-1, homocysteine, and fibrinogen levels and race/ethnicity in women without cardiovascular disease. Am J Cardiol 2007;99:1246-51.

32. Albert MA, Pare G, Morris A, et al. Candidate genetic variants in the fibrinogen, methylenetetrahydrofolate reductase, and intercellular adhesion molecule-1 genes and plasma levels of fibrinogen, homocysteine, and intercellular adhesion molecule-1 among various race/ethnic groups: data from the Women's Genome Health Study. Am Heart J 2009;157:777-83.

33. Zhang Y, Zhu CG, Guo YL, et al. Higher fibrinogen level is independently linked with the presence and severity of new-onset coronary atherosclerosis among Han Chinese population. PLoS One 2014;9:e113460. 